\title{
Supplementation of standard antibiotic therapy with oral probiotics for bacterial vaginosis and aerobic vaginitis: a randomised, double-blind, placebo- controlled trial
}

\author{
Piotr B. Heczko ${ }^{1}$, Anna Tomusiak ${ }^{1}$ Paweł Adamski², Artur J. Jakimiuk ${ }^{3,4}$, Grzegorz Stefański ${ }^{5}$,
} Aleksandra Mikołajczyk-Cichońska ${ }^{5}$, Magdalena Suda-Szczurek ${ }^{5}$ and Magdalena Strus ${ }^{*}$

\begin{abstract}
Background: This multicentre, randomised, double-blind, placebo-controlled trial was performed to determine whether the use of oral probiotic preparation $\left(\mathrm{prOVag}^{\oplus}\right.$ ) containing three Lactobacillus strains together with standard metronidazole treatment and also targeted antibiotic treatment (following the failure of metronidazole therapy) could reduce the recurrence rates of bacterial vaginosis (BV) and aerobic vaginitis (AV).

Methods: Patients at private gynaecological clinics in Poland with histories of recurrent BV/AV and current symptoms were randomly allocated to receive metronidazole and probiotic or placebo, and assessed monthly on visits II and III-V. The total number of study visits was 5-6 (I, II, II bis - if applicable, III, IV, V). One probiotic or placebo capsule was administered with metronidazole/targeted antibiotic twice daily for 10 days; during follow up, patients took one capsule daily for 10 days perimenstrually. Clinical examination and vaginal swabbing were performed at each visit. Primary outcomes were clinical or microbiological BV/AV recurrence and probiotic safety. Secondary outcomes were vaginal $\mathrm{pH}$, Nugent score, and Lactobacillus counts in the vaginal microbiota. Safety analysis was performed in 578 (probiotic, $n=285$; placebo, $n=293$ ) 18-50-year-old women who were randomised.

Results: BV/AV was confirmed microbiologically in 241 (probiotic, $n=118$; placebo, $n=123$ ) participants, who continued the trial. Data from 154 (probiotic, $n=73$; placebo, $n=81$ ) participants who completed the study were analysed to determine the efficacy of prOVag. Additional analyses included 37 (probiotic, $n=22$; placebo, $n=15$ ) participants who received targeted antibiotics and probiotics or placebo. prOVag lengthened the time to clinical relapse of BV/AV symptoms up to $51 \%(p<0.05)$ compared with placebo; AV relapse was delayed by up to $76 \%$ $(p<0.05)$. Probiotic use also reduced and maintained low vaginal $\mathrm{pH}$ and Nugent score, and increased vaginal Lactobacillus counts following standard treatment.
\end{abstract}

Conclusion: This study demonstrated that oral probiotics lengthened remission in patients with recurrent BV/AV and improved clinical and microbiological parameters.

Trial registration: NCT01993524; 20 November 2013.

Keywords: Probiotics, Lactobacillus, Bacterial vaginosis, Aerobic vaginitis

\footnotetext{
* Correspondence: mbstrus@cyf-kr.edu.pl

${ }^{1}$ Chair of Microbiology, Jagiellonian University Medical College, Krakow,

Poland

Full list of author information is available at the end of the article
} 


\section{Background}

Bacterial vaginosis (BV) is the most common vaginal infection in women of reproductive age [1], caused by overgrowth of anaerobic bacteria with reductions in Lactobacillus populations in the vagina [2]. Aerobic vaginitis (AV), referred to as 'intermediate flora', is related to the suppression of lactobacilli by various aerobic bacteria [3-5]. AV is often found in patients treated unsuccessfully for BV with antibiotics [6]. The standard therapy for BV is oral metronidazole, vaginal clindamycin cream, or metronidazole gel [7], whereas targeted therapy according to an individual's antibiotic resistance is recommended for AV [6]. Treatment success rates of about $50 \%$ have been reported for $\mathrm{BV}$ and $\mathrm{AV}$, and recurrence rates can exceed $50 \%$ within 6-12 months after treatment cessation [8, 9]. Most proposed nonantibiotic therapies for BV are vaginal or oral probiotics, which aim to restore the normal vaginal microbiota [2]. Several trials evaluated the use of combined metronidazole and/or clindamycin therapy and vaginal probiotics to prevent BV recurrence. In a study by Larsson et al. [10], BV was treated with clindamycin cream and, subsequently, a vaginal probiotic containing L.gasseri and L.rhamnosus or placebo. Use of the probiotic contributed to prevention of relapse (hazard ratio $=0.73$ ). Marcone et al. [11] treated 24 women with BV initially with oral metronidazole, and then with vaginal doses of L.rhamnosus for 6 months. Efficacy was evaluated solely using microbiological data. Abnormal vaginal microflora was found in $31 \%$ subjects after 12 months compared with $9 \%$ directly after metronidazole treatment in control group (significant increase in the number of women with abnormal flora over time). The same follow-up study showed no statistically significant difference in vaginal microflora in women from probiotic group (9 \% during 12 months follow-up vs. $4 \%$ directly after metronidazole). Ya et al. [12] conducted a study, in which 58/120 women with histories of recurrent BV were given vaginally L.rhamnosus, L.acidophilus, and S.thermophilus daily for 2 months, while 62 women received placebo. After 11 months, study subjects were interviewed by telephone about BV symptoms; $15.8 \%$ of women who used the probiotic and $45 \%$ of control subjects reported symptoms of BV. Delia et al. [13] published a report on a 3-month administration of a vaginal probiotic containing L.acidophilus or the same probiotic plus oral L.paracasei to women with suspected BV or BV confirmed by Amsel's criteria. The authors concluded that the vaginal probiotic reduced $\mathrm{pH}$ and improved other parameters, and that the adjuvant oral probiotic sustained this effect for 6 months. Results of a recent double-blind, randomised, placebocontrolled clinical trial [14] on treatment of BV by oral application of two probiotic bacteria described earlier by Reid [15] show restoration of vaginal microbiota balance within 44 days in $61.5 \%$ of patients receiving the probiotic, compared with nearly $27 \%$ of the placebo group. Unfortunately, the diagnosis of vaginal microbiota balance was based only on a presumptive detection of lactobacilli based on four phenotypic criteria, one of which was misleading because a considerable proportion of Lactobacillus species produce catalase [16]. As stated by Hay, advances in our understanding of the pathogenesis of bacterial vaginosis allow the opportunity to improve treatments to prevent recurrence, which may require a combination of modalities [17]. As emphasised in a recent Cochrane review [18], large, randomised, placebo-controlled trials with standardised outcomes are needed to confirm the efficacy of probiotic/antibiotic treatment for BV. The efficacy of such combination treatment for AV has not been examined.

Thus, the aim of this study was to investigate whether the use of oral probiotic preparation prOVag together with standard treatment for BV or AV could reduce the recurrence rates, as per clinical and microbiological criteria, in comparison with standard treatment alone. prOVag is a preparation containing strains of three lactic acid bacteria: L.fermentum 57A, L.plantarum 57B, and L.gasseri 57C. All strains were isolated from a vaginal swab taken from the same healthy woman aged 26 years not using antibiotics for the last 3 months. The strains possessed high co-aggregating abilities and naturally occurred as a triad strains complex. Their species designation was confirmed by PCR for $16 \mathrm{~S}$ RNA using species-specific primers and they were identified using both PFGE and MLST methods to distinguish them from other strains in materials taken during clinical study. They have been deposited in the internationally recognized collection and covered with a patent. They had been selected for commercial use on the basis of high adherence ability to human Caco-2 enterocytes and A431 vaginal cell lines, ability of selected vaginal pathogens adhesion reduction already adhered to these lines and a broad antagonistic properties exerted against G.vaginalis, S.agalactiae, P.bivia, S.aureus, E.faecalis, C.difficile and uropathogenic E.coli. L.gasseri $57 \mathrm{C}$ produces hydrogen peroxide. All of them are resistant to metronidazole and ciprofloxacin. L.fermentum 57A and L.plantarum 57B are vancomycin-resistant while L.gasseri $57 \mathrm{C}$ is sensitive to vancomycin. The strains carry no extrachromosomal DNA elements able to transmit resistance to antibiotics, and they are resistant to gastric juice with pepsin at $\mathrm{pH} 2.5$, to bile salts and to spermicides like as nonoxynol-9.

\section{Methods}

This multicentre, randomised, double-blind, placebocontrolled, parallel-group study was conducted between March 2009 and February 2012. Nine private outpatient 
gynaecological clinics in Poland recruited the patients. The trial received ethical approval from the independent ethics committee of the Silesia Medical Chamber, relevant to the study coordinator (no. 4/2009, 2 February 2009). The study protocol number is PB-DM/SBKprOVag2-01/08. All participants provided written informed consent.

The sample size was calculated with the assumption that the product would be $\geq 50 \%$ more efficient than placebo [19]. Previous studies have documented recurrence rates of $\sim 40 \%$ within 3 months following the end of treatment [20, 21]; thus, this recurrence rate was expected in subjects on placebo and the probiotic product was expected to reduce the recurrence rate to $20 \%$. The sample size was calculated with the pwr procedure in the $\mathrm{R}$ software package (version 2.15.0; $\mathrm{R}$ Development Core Team, 2012). Under these assumptions, the minimum sample size was determined to be 148 patients (74 per group) who successfully complete the clinical study. In recognition of several factors that may reduce the sample size, the screening group was increased to about 600 participants (probiotic group, $n=285$; placebo group, $n=293$ ).

Eligible participants were 18-50-year-old European descent women who menstruated regularly and had histories of recurrent BV. Table 1 lists the inclusion and exclusion criteria. Participants were asked not to use any new intimate hygienic product or vaginal douche during the trial. Participants recorded safety information in 'patient's diaries', which an investigator analysed and documented at each follow-up visit.

A clinician examined all participants, recorded medical histories and clinical signs confirming a vaginal bacterial condition (BV/AV), and collected high vaginal swabs for further microbiological analysis performed in the central laboratory. Clinical examination and vaginal swabbing were performed at each visit as well as vaginal $\mathrm{pH}$ value that was measured by the investigator with $\mathrm{pH}$ indicator (Merck, range: 4.0-7.0). One vaginal swab from each participant was used to estimate Nugent scores [22]. The second vaginal swab was transferred from transport medium (Amies, Deltalab, Barcelona, Spain) to $1 \mathrm{ml}$ Man, Rogosa, and Sharpes (MRS) broth (Difco Laboratories Inc., Detroit, MI,USA) and agitated for $1 \mathrm{~min}$. Serial decimal dilutions in the same broth were then made, and $100-\mu \mathrm{l}$ aliquots were plated on standard media for cultivation of aerobic and anaerobic bacteria and Candida yeasts. Bacteria were cultivated at $37{ }^{\circ} \mathrm{C}$ in aerobic or aerobic atmosphere for 24 or 48 hours. G.vaginalis isolates were identified on the basis of haemolysis on human blood agar, Gram-stained morphology showing typical Gram-negative rods, and negative catalase production. Streptococcus agalactiae, S.bovis, Escherichia coli, Enterococcus spp., and strict anaerobes such as Prevotella bivia were identified using standard phenotypic methods (API STREP, API STAPH, API 20E, and API 20A; bioMerieux). Only those microbial species or genera, found in cultures from vaginal swabs in numbers exceeding $10^{5}$ c.f.u. per swab, were regarded as related to vaginal infection [23].

G. vaginalis strains were tested for resistance to metronidazole and clindamycin using E-tests (bioMerieux). The resistance of common aerobic vaginal pathogens was tested using EUCAST disk diffusion method [24] for the following: azithromycin, doxycycline, amoxicillin, amoxicillin with clavulanic acid.

All eligible women were given at I visit a standard treatment $(500 \mathrm{mg}$ oral metronidazole twice daily for 7 days) and were randomly assigned to one of two study arms (1:1) (using block randomisation with a block size of 12 and equal group ratios) to receive probiotic or placebo twice daily for 10 days. At II visit (about 14 days after probiotic or placebo treatment cessation), participants were checked for symptoms and signs of vaginal infection and their vaginal swabs were analyzed for the presence of pathogens. If both, symptoms and G.vaginalis, were still present, metronidazole-resistant G.vaginalis BV infection was confirmed microbiologically and the patients were given oral clindamycin. When the infection signs and aerobic bacteria were found, AV diagnosis was made and an antibiotic indicated by individual susceptibility

Table 1 Inclusion and exclusion criteria applied in the study

\begin{tabular}{ll}
\hline Inclusion & Exclusion \\
\hline $\begin{array}{l}\text { At least three of the following BV symptoms, according to Amsel's } \\
\text { criteria [13]: homogenous vaginal discharge, vaginal pH }>4.5, \text { positive }\end{array}$ & $\begin{array}{l}\text { Women were ineligible if they were pregnant or breastfeeding; had } \\
\text { amine test findings, and presence of clue cells in a wet smear observed }\end{array}$ \\
$\begin{array}{ll}\text { under microscopy. } & \text { other antibiotics, Candida vaginitis, bleeding from the genital tract of } \\
\text { unknown aetiology, any pathology of the reproductive organs, congenital }\end{array}$ \\
$\begin{array}{ll}\text { or acquired immunodeficiency, diabetes, mental illness, or neoplastic } \\
\text { disease; used mechanical contraceptives, such as diaphragms, intrauterine } \\
\text { contraceptive inserts, or hormonal vaginal rings; had used oral hormonal } \\
\text { preparations or vaginal oestrogens in the reproductive period; were using } \\
\text { another oral and/or vaginal probiotic at the time of assessment for } \\
\text { inclusion in the study; had participated in another clinical study within the } \\
\text { previous 30 days; were receiving antibiotic therapy for another reason; or } \\
\text { were scheduled for surgery or hospitalisation. }\end{array}$ \\
\hline
\end{tabular}


testing was used. Antibiotic was taken together with probiotic or placebo twice daily for 10 days.

Participants without signs of the infection during II visit were given only the probiotic or placebo once daily for 10 days in the peri-menstrual period (starting from 18 to 22 day of menstrual cycle) for the next 3 months. Participants with targeted antibiotic ordered on visit II were to come to the visit II bis, and if secondary treatment was successful they were to proceed for the next 3 months in the same manner as described above. Visits III-V occurred for all patients within $\sim 7$ days after completion of each menstrual period.

The probiotic used was a commercially available preparation $\left(\mathrm{prOVag}^{\circ}\right)$ provided by the study sponsor (IBSS BIOMED S.A.,Krakow,Poland) containing a mixture of three viable strains: L.gasseri 57C, L.fermentum 57A, and L.plantarum 57B in a total number of $\geq 10^{8}$ c.f.u. Placebo looked identical but contained excipients only. Metronidazole and targeted antibiotics were licensed products provided by the study sponsor.

Recurrence of BV/AV at visits III-V was the study endpoint which means that patients diagnosed with a clinical relapse of BV/AV at visits III-V ended their participation in the study on the day of the visit when the diagnosis was made. However, if the reoccurrence of BV/AV was diagnosed at visit II (as a consequence of not efficient treatment), the patient stayed in the study. Nonetheless BV/AV diagnosis at visit II bis meant withdrawal of the study for the participant. The numbers and types of adverse events and serious adverse events were also assessed. The reduced probability of recurrent bacterial vaginal infection, confirmed by clinical symptoms and/or microbiological results, was the parameter used to assess primary efficacy. Secondary parameters were normalisation and maintenance of vaginal $\mathrm{pH}$, Nugent score, and presence of $>10^{5}$ c.f.u. of Lactobacillus in the vaginal flora per swab.

Data obtained at visits III-V were compared with those obtained at visit II. The time to recurrence was measured from the visit on which a participant showed no BV/AV symptom: visit II for participants on metronidazole treatment and the visit IIbis for participants treated with metronidazole and targeted antibiotics.

Statistical analysis were performed with $\mathrm{JMP}^{\circ}$ software (version 7.0.1, SAS Institute Inc., Cory, NC, USA) and the R2.7.2 software package (R Foundation for Statistical Computing). Quantitative variables were compared between groups using Student's $t$-test, or non-parametric (Mann-Whitney or Wilcoxon) tests in the case of nonnormal distribution. For comparisons of more than two groups, analysis of variance with a post-hoc Tukey's test was used. The non-parametric equivalents of these tests (Kruskal-Wallis and Steel-Dwass tests) were used to analyse variables that did not comply with the assumptions of analysis of variance. Data obtained at multiple visits were compared using parametric (Student's $t$-test for matched pairs) or non-parametric (Wilcoxon signed rank) matched-pairs tests. Categorical variables were analysed using frequency (chi-squared or likelihood ratio) tests. The significance level for statistical analysis was set at $p<0.05$.

\section{Results}

The safety analysis included 578 participants in the intent-to-treat population, 241 (prOVag group, $n=118$; placebo group, $n=123$ ) of whom complied with the recommended regimen and completed treatment (i.e.used $\geq 20$ capsules). No participant was excluded from the study due to an adverse event, and no serious adverse event related to the use of the probiotic product occurred. In total, there were 431 adverse events in 160 participants, which accounts for $28 \%$ of the population for which the safety analysis was carried out (ITT); prOVag was used by 77 of these participants. There were no significant differences between the total numbers of adverse events reported in either of the study groups. Over half of all reported adverse events were considered to be unrelated to the use of the investigational product. Most of the reported adverse events concerned disorders of the gastrointestinal system, reproductive system and breasts. Main disorders of the gastrointestinal system included events possibly related to the use of metronidazole. The analysis of the time of their occurrence demonstrated that they were mainly reported at visit II, i.e. directly after the period when the participant used metronidazole. The statistical analysis of data did not demonstrate a significant correlation between using the investigational product by the participants and the occurrence of adverse events.

Table 2 presents the participants' baseline characteristics. No significant difference in these characteristics between groups was found. Safety analysis was performed on the group of 578 participants who used at least one capsule of tested product (intent to treat-ITT) and the efficacy analysis was performed on the population who completed the full cycle of visits $(n=154$, per protocolPP). Figure 1 shows a diagram of participant flow.

Relapse rates based on clinical symptoms didn't show any significant differences between placebo and prOVag groups. However, the average times to relapse were 47.3 [standard deviation $(\mathrm{SD})=26.98$ ] days in the placebo group and 71.4 $(\mathrm{SD}=37.51)$ days in the prOVag group. This interval, which was up to $51 \%$ longer in the prOVag group, differed significantly between placebo-treated and control patients $(t=2.606, p=0.0125$; Fig. 2).

Fifty one participants showed BV/AV clinical relapse, 37 of them (prOVag group, $n=22$; placebo group, $n=15$ ) received secondary targeted treatment since either aerobic 
Table 2 Baseline characteristics ${ }^{a}$ of patients in the intent-to-treat (ITT; $n=578)$ and per-protocol (PP; $n=154)$ populations

\begin{tabular}{|c|c|c|c|}
\hline Characteristic & Placebo & Probiotic & $p$ \\
\hline Hormonal treatment ITT & 15 & 20 & 0.3388 \\
\hline Hormonal treatment PP & 3 & 5 & 0.3798 \\
\hline Contraception ITT & 89 & 91 & 0.6866 \\
\hline Contraception PP & 26 & 20 & 0.5244 \\
\hline Contraceptive tablets ITT & 66 & 71 & 0.4999 \\
\hline Contraceptive tablets PP & 16 & 13 & 0.8095 \\
\hline Transdermal contraception ITT & 10 & 6 & 0.3380 \\
\hline Transdermal contraception PP & 2 & 0 & 0.2047 \\
\hline Contraception condom ITT & 10 & 8 & 0.6750 \\
\hline Contraception condom PP & 5 & 4 & 0.9480 \\
\hline Contraception other ITT & 4 & 6 & 0.4951 \\
\hline Contraception other PP & 3 & 3 & 0.9420 \\
\hline Sexually active ITT & 270 & 266 & 0.5838 \\
\hline Sexually active PP & 76 & 70 & 0.5646 \\
\hline More than one sexual partner ITT & 1 & 1 & 0.9844 \\
\hline More than one sexual partner PP & 0 & 0 & \\
\hline Smoking ITT & 46 & 32 & 0.1120 \\
\hline Smoking PP & 14 & 12 & 0.8888 \\
\hline Vaginal douching ITT & 2 & 3 & 0.6310 \\
\hline Vaginal douching PP & 1 & 1 & 0.9411 \\
\hline \multicolumn{4}{|l|}{ Complaints at first visit (PP) } \\
\hline Vaginal itching & 7 & 6 & 0.9249 \\
\hline Vaginal burning & 14 & 14 & 0.7301 \\
\hline Vaginal pain & 1 & 1 & 0.9410 \\
\hline Vaginal discharge & 79 & 70 & 0.5663 \\
\hline Pelvic pain & 2 & 1 & 0.6221 \\
\hline
\end{tabular}

Conditions reported by investigators at first visit (PP)

$\begin{array}{llll}\text { Vaginal erythema } & 0 & 3 & 0.0354 \\ \text { Palpation tenderness } & 1 & 1 & 0.9624 \\ \text { Homogenous vaginal discharge } & 78 & 71 & 0.9260 \\ \text { Clue cell presence } & 79 & 71 & 0.9160 \\ \text { Positive amine test } & 78 & 72 & 0.3632 \\ \text { BV (Amsel's criteria [13]) } & 80 & 72 & 0.2559 \\ \text { Vulvovaginal candidiasis } & 0 & 1 & 0.2206\end{array}$

Microbial species cultured from vaginal swabs collected at first visit ${ }^{\mathrm{b}}(\mathrm{PP})$

$\begin{array}{llll}\text { Gardnerella vaginalis } & 46 & 52 & 0.0628 \\ \text { Streptococcus agalactiae } & 17 & 15 & 0.9465 \\ \text { Escherichia coli } & 5 & 3 & 0.5646 \\ \text { Enterococcus spp. } & 0 & 1 & 0.2206 \\ \text { Candida albicans } & 2 & 0 & 0.1076 \\ \text { Lactobacillus spp. } & 48 & 44 & 0.8980\end{array}$

${ }^{a}$ Analyses were not carried for age, sex, or race, as only Caucasian women aged 18-50 years were included

${ }^{\mathrm{b}}$ Only populations exceeding $10^{5}$ organisms per swab were counted bacteria or metronidazole-resistant G.vaginalis were isolated. The intervals between the end of treatment and relapse in these patients were similar to the entire group: the time to relapse was up to $76 \%$ longer in the prOVag group ( $72.4 \pm 37.87$ days) than in the placebo group (41.2 \pm 24.86 days; $t=2.983324, p=0.0053$; Fig. 3 ).

Analysis of bacteriological data collected at visit I demonstrated that clinical symptoms were related most frequently to the presence of G.vaginalis ( $n=98$ patients), followed by S.agalactiae $(n=32)$, E.coli $(n=8)$, Enterococcus spp. $(n=1)$, and other bacteria (e.g. P.bivia, S.bovis).

The relapse rate diagnosed on the basis of microbiological criteria (presence of $>10^{5}$ c.f.u./swab of any bacterial species potentially related to BV/AV at visits III-V), occurred in 71 patients [prOVag group, $n=33(45.2 \%)$; placebo group, $n=38(47.0 \%)$ ]. This difference approached statistical significance $\left(\chi^{2}=4.883, p=0.0870\right)$.

In both study groups, the incidence of abnormal vaginal microbiota (G.vaginalis or aerobic pathogens) decreased at subsequent visits. The incidence of microbiologically confirmed BV/AV decreased significantly and progressively from visit III to visit V in the prOVag group, whereas significant reductions were observed only through visit IV in the placebo group. Thus, significantly fewer patients in the prOVag group had microbiologically confirmed BV/AV at visit $\mathrm{V}$, compared with the placebo group $\left(G^{2}=3.9706, p=0.04632\right.$; Fig. 4$)$.

Because the interval between the end of treatment and relapse of microbiologically confirmed BV/AV was not normally distributed ( $W=0.868216, p<0.00001$ ), the non-parametric Wilcoxon test was used. The interval in which no abnormal microbial flora was detected in the vagina was longer in the prOVag group (35.0 days; $\mathrm{GK}=54, \mathrm{DK}=28$ ) than in the placebo group (28.5 days; $\mathrm{GK}=49, \mathrm{DK}=21$ ), but this difference was not significant $(z=0.60352, p=0.5426$; Fig. 5).

Mean vaginal $\mathrm{pH}$ values decreased between all visits in the prOVag group, whereas slight increases in $\mathrm{pH}$ values were observed between visits III and V, and IV and V visits in the placebo group. Between visits IV and V, a mean increase of 0.18 units in the placebo group, and a mean decrease of 0.03 units in the prOVag group were observed; differences in $\mathrm{pH}$ changes between groups were significant (Table 3).

Changes in Nugent scores were not normally distributed ( $W=0.9047 ; p<0.0001)$ and were analysed using KruskalWallis test. The scores differed significantly between groups throughout the study period $\left(H_{\text {placebo }}=115.874\right.$, $\left.p_{\text {placebo }}<0.0001 ; \quad H_{\text {provag }}=87.392, \quad p_{\text {provag }}<0.0001\right)$. However, post-hoc Steel tests demonstrated that Nugent scores obtained at visit I did not differ significantly from those obtained at visits III-V in either group. As in the analysis of $\mathrm{pH}$, matched-pair tests were used to 


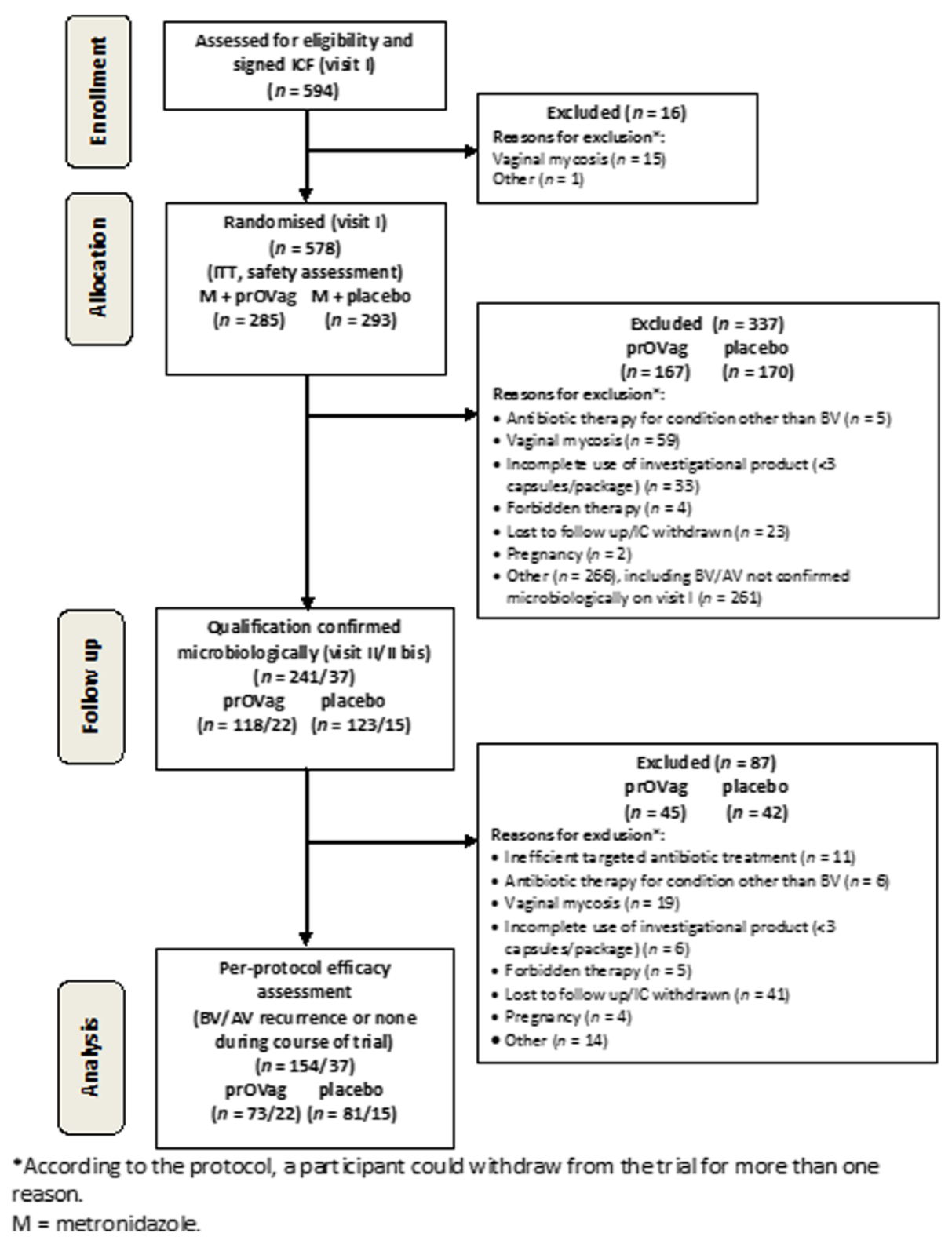

Fig. 1 Participant flow

eliminate the effects of individual differences between patients. In both groups, the Nugent scores tended to decrease over time; only differences between visits I and III, and III and IV were significant in the placebo, whereas the differences between visits I and III, I and IV, IV and $\mathrm{V}$ were significant in the prOVag group (Table 4). After the exclusion of patients in whom no change was observed, the probability of a reduction in the Nugent score between visits IV and V was significantly higher in the prOVag than in the placebo group $\left(x^{2}=3.937, p=0.0472\right)$.

Total counts of vaginal Lactobacillus increased in both groups during the study period $\left(f_{\text {placebo }}=7.0664\right.$, $\left.p_{\text {placebo }}<0.0001 ; \quad f_{\text {provag }}=8.9136, \quad p_{\text {provag }}<0.0001\right)$. Post-hoc Tukey's tests revealed that a significant increase in Lactobacillus numbers vs. visit I was observed an average of one menstrual cycle earlier (visit III) in the prOVag (Fig. 6) than in the placebo group (visit IV; Fig. 7).

\section{Discussion}

In the present study, we examined effects of the standard antibiotic treatment together with oral probiotic intake on recurrences of BV observed as clinical symptoms of vaginal infection and disturbances in the composition of vaginal microbiota. The number of subjects enrolled, 


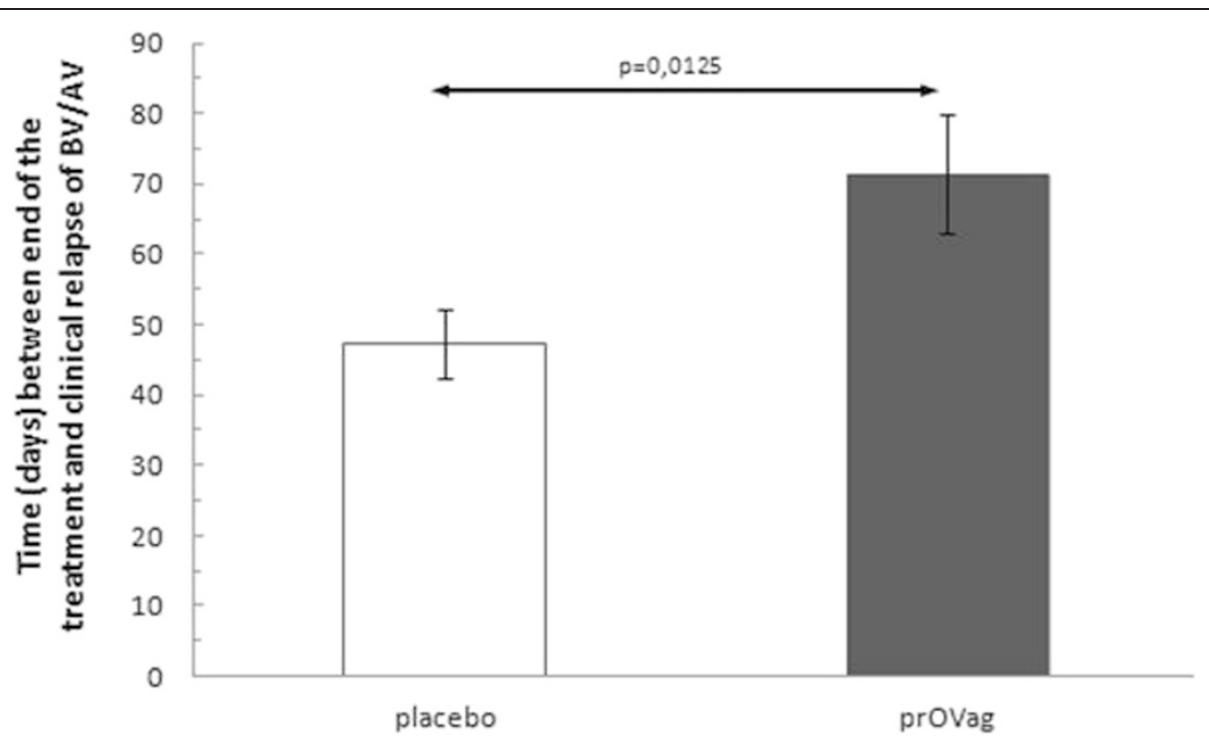

Fig. 2 Interval between end of treatment and appearance of clinical relapse in patients on standard therapy taking prOVag or placebo

methods used to evaluate vaginosis, and results obtained address the calls for well-designed, placebo-controlled clinical studies involving large patient groups to examine the use of probiotics to treat BV that have been put forward by the authors of reviews and meta-analyses [18].

Our study protocol focused on demonstrating that the use of the prOVag probiotic preparation simultaneously with standard treatment for BV reduced the rate of relapses, assessed using clinical and microbiological criteria, versus the standard treatment alone.

This study was conducted in several clinical centers located in southern Poland using the same protocol. In order to ensure the study consistency with the protocol and conform to Good Clinical Practices (ICH-GCP), the whole course of the trial was monitored by third party monitoring entities (CRO), which guaranteed protection of participants' rights, their safety, and completeness, reliability, and quality of the obtained data. Moreover the study was constantly under supervision of Medical and Microbiological Experts. Microbiological analyses were performed in one central laboratory possessing accreditation ISO certificate $\mathrm{Nr} A B 1428$ for performing tests for vaginal infections. Obviously, more clinical studies done in different countries would be desirable to confirm and validate clinical efficacy of prOVag in women with various types of the vaginal microbiota alterations related to BV or AV [25].

Our trial involved a much larger number of participants than did previous studies. In addition, no previously published study used both widely accepted clinical

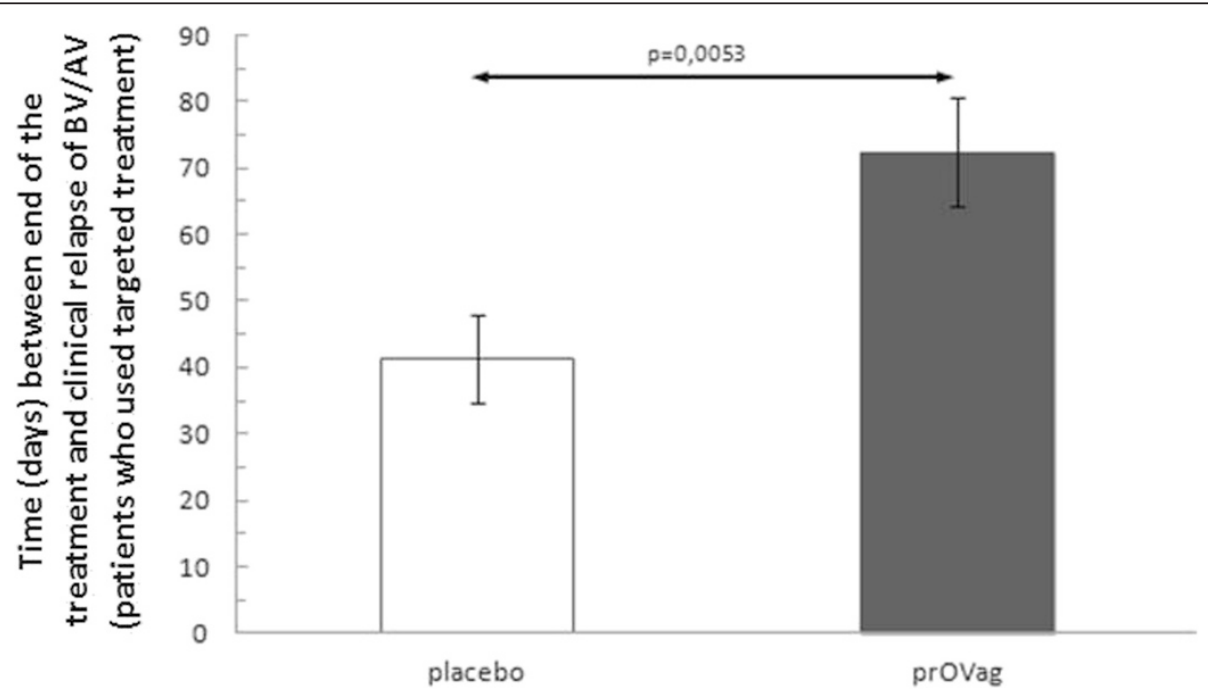

Fig. 3 Interval between end of treatment and clinical relapse in patients receiving targeted antibiotics and prOVag or placebo 


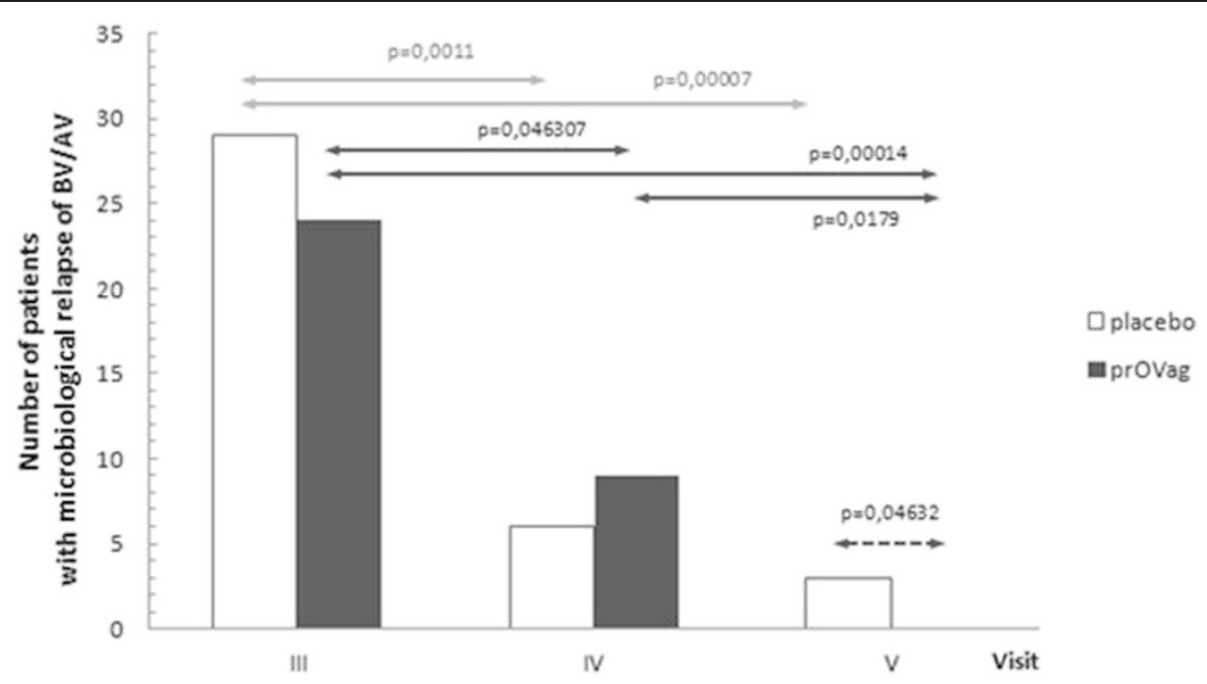

Fig. 4 Number of patients with microbiological relapse detected at consecutive visits

(Amsel) and microbiological (Nugent score and pathogen count vs. Lactobacillus count) criteria. Our analysis demonstrated that the use of prOVag achieved a significantly longer interval between cure and the relapse of clinical symptoms of BV, compared with placebo. Because the criteria used for microbiological assessment are much stricter than those for clinical assessment, no such effect was observed for the interval between cure and microbiological relapse (i.e. redetection of abnormal vaginal microflora). Microbiological analysis of vaginal smears was used to demonstrate colonisation of the vaginal epithelium by pathogens, expressed as abnormal composition of vaginal microbiota. This definition of colonisation implies that it precedes the onset of $\mathrm{BV}$ symptoms observed by patients and physicians, and explains the difference observed in the times to microbiological and clinical relapse.

The presented study also included women with AV. To date, no other study has evaluated the effect of oral probiotics on the course of AV. We found that the use of prOVag significantly delayed the clinical relapse of AV symptoms by an average of 31 days (up to $76 \%$ ) in patients who used a targeted antibiotic, i.e. those in whom pathogens responsible for $\mathrm{AV}$ ( $S$. agalactiae, $E$. coli, E. faecalis) were detected, compared with placebo.

The analysis of changes in vaginal $\mathrm{pH}$ and Nugent score values between the first and fifth visit confirmed the efficacy of prOVag compared with placebo. Women using prOVag showed continuous improvement in these parameters from the first to fifth visits. Changes in

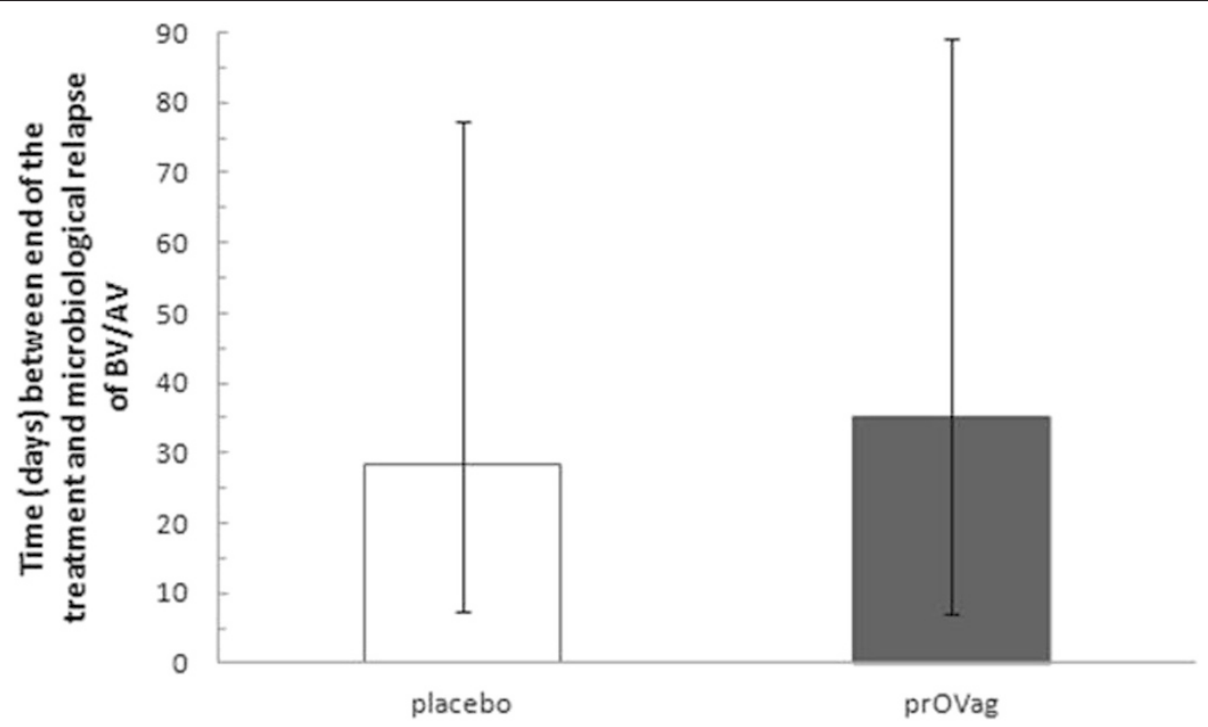

Fig. 5 Interval between end of treatment and microbiological relapse in patients receiving antibiotics and taking provag or placebo 
Table 3 Mean changes in vaginal pH between visits

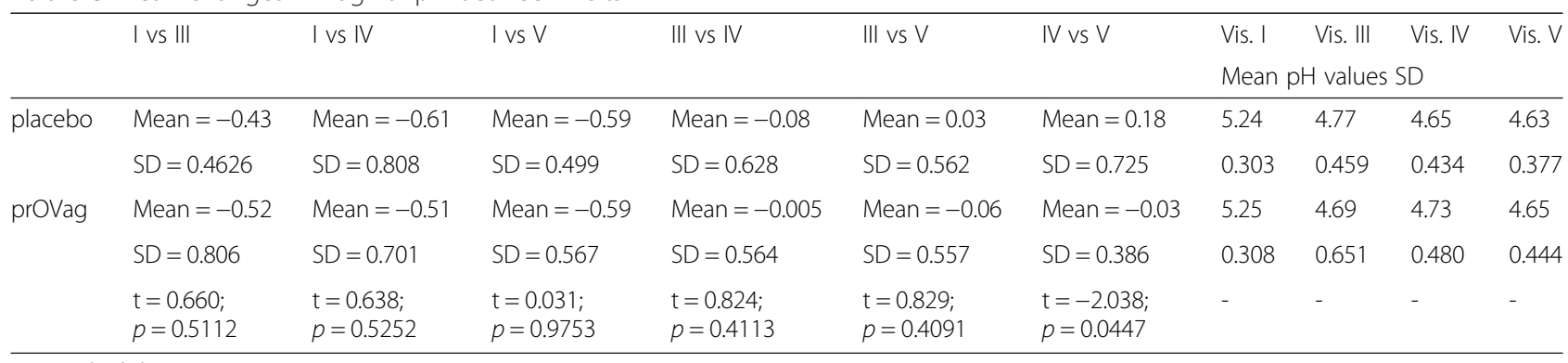

SD standard deviation

vaginal microflora were associated with increased Lactobacillus counts, which decreased the $\mathrm{pH}$ of the vaginal environment and inhibited the growth of pathogens. The population of Lactobacillus was restored significantly faster after standard treatment in women using prOVag than in the placebo group. Similar results were obtained in our previous open-label study of the use of prOVag by women with intermediate/abnormal vaginal flora and no clinical symptoms [26].

Thus, the use of prOVag contributes significantly to the maintenance and prolongation of treatment outcomes achieved with targeted antibiotics in patients with $\mathrm{AV} / \mathrm{BV}$, selected on the resistance of vaginal pathogens to chemotherapeutics. No report of the possible use of probiotic preparations to enhance and prolong the treatment outcome of AV achieved with different antibiotics has been published previously. The same effect, as a total novelty, was achieved in women treated with targeted oral clindamycin due to the resistance of

Table 4 Changes in Nugent scores at subsequent visits

\begin{tabular}{|c|c|c|c|c|c|}
\hline & Visit III & Visit IV & Visit V & Mean & SD \\
\hline \multicolumn{6}{|c|}{ Placebo } \\
\hline \multirow[t]{2}{*}{ Visit I } & $H=702.0 \downarrow$ & $H=770.0 \downarrow$ & $H=780.0 \downarrow$ & 6.3 & 1.68 \\
\hline & $p<0.0001$ & $p<0.0001$ & $p<0.0001$ & & \\
\hline \multirow[t]{2}{*}{ Visit III } & - & $H=150.0 \downarrow$ & $H=200.0 \downarrow$ & 3.0 & 2.54 \\
\hline & & $p=0.0037$ & $p=0.0083$ & & \\
\hline \multirow[t]{2}{*}{ Visit IV } & - & - & $H=23.0 \downarrow$ & 2.1 & 2.01 \\
\hline & & & $p=0.716$ & & \\
\hline Visit V & - & - & - & 1.7 & 2.18 \\
\hline \multicolumn{6}{|c|}{ prOVag } \\
\hline \multirow[t]{2}{*}{ Visit I } & $H=632.0 \downarrow$ & $H=642.5 \downarrow$ & $H=678.0 \downarrow$ & 6.2 & 1.92 \\
\hline & $p<0.0001$ & $p<0.0001$ & $p<0.0001$ & & \\
\hline \multirow[t]{2}{*}{ Visit III } & - & $\mathrm{H}=33.5 \downarrow$ & $H=106.00 \downarrow$ & 2.9 & 2.58 \\
\hline & & $p=0.4484$ & $p=0.0726$ & & \\
\hline \multirow[t]{2}{*}{ Visit IV } & - & - & $H=131.0 \downarrow$ & 2.4 & 2.50 \\
\hline & & & $p=0.0201$ & & \\
\hline Visit V & - & - & - & 1.8 & 2.52 \\
\hline
\end{tabular}

G.vaginalis strains to routinely used metronidazole. Because the number of resistant G.vaginalis strains has increased recently [27], the possibility of using prOVag in such cases offers considerable benefits for a growing number of patients. Thus, the effect of prOVag is independent of the administered antibiotic and relies on efficient and persistent restoration of the normal composition of vaginal microflora, which naturally protects the vaginal epithelium from colonisation by pathogenic factors [28].

From clinical point of view, since metronidazole, the most commonly prescribed antibiotic to treat BV, causes only short-term suppression of the G.vaginalis populations in the vagina as revealed recently by Meyer et al. [29], the combined therapy with this antibiotic plus prOVag can successfully eliminate anaerobic bacterial pathogens responsible for $\mathrm{BV}$, thereby delaying or preventing the relapse of vaginal inflammation. Their paper in fact augments importance of the combined treatment with metronidazole plus oral probiotic in long-term prevention of recurrent BV.

Although Lactobacillus strains used in prOVag are susceptible to clindamycin in vitro, their uninterrupted application throughout the study has sustained vaginal colonization. In fact, little is known about bactericidal effect of clindamycin against lactobacilli. Aroutcheva et al. found that the mean MIC values for studied lactobacilli was as high as $1000 \mathrm{ug} / \mathrm{ml}$ and stated that only high concentrations of clindamycin achieved in the vagina might be inhibitory for Lactobacillus. Clindamycin was rapidly eliminated from the vagina, within 3-8 days, after local administration [30]. Results shown by Eriksson et al. indicate that local treatment with probiotic lactobacilli could be problematic if carried out within 5 days after cessation of clindamycin treatment, but this is not in the case of the oral application [31]. Moreover, we have demonstrated before that maximal vaginal colonization with the prOVag strains was recorded between 31st and 60th day after the initiation of the treatment [26]. Most probably, an efficient colonization of the colon and rectum creates dense populations of the lactobacilli, which then constantly move to vagina. On the 


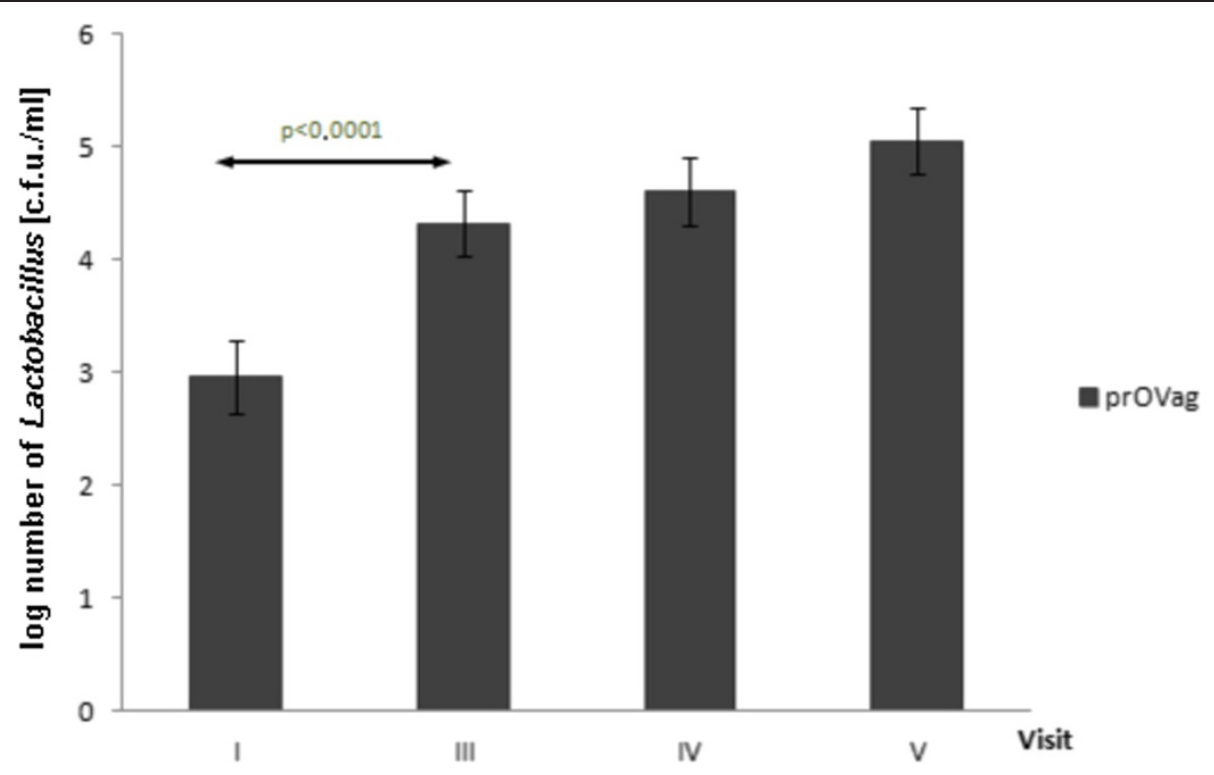

Fig. 6 Effect of prOVag application on total numbers of vaginal lactobacilli during consecutive visits

other hand, our lactobacilli were resistant to metronidazole and thus oral application of this drug should not influence their populations in rectum and vagina which is quite important from the practical point of view in the light of the recent findings reported by Mayer et al. [29].

Vaginal delivery of the probiotic strains via oral/anal route seems to be well documented in the light of the papers published by Reid's group and us [26, 32]. It may be therefore proposed to consider probiotic supplementation of the antibiotic therapy for recurrent BV as a new adjuvant treatment for preventing recurrences.

\section{Conclusions}

The analysis of efficacy parameters demonstrated that prOVag significantly lengthened the time to relapse of BV/AV clinical symptoms compared with placebo. In addition, the effect of prOVag was more powerful in patients using a targeted antibiotic (i.e.those diagnosed with $\mathrm{AV}$ and/or metronidazole-resistant G.vaginalis) than in the per-protocol population. In such cases, prOVag lengthened the time to BV relapse by as much as $76 \%$. We also found that the use of prOVag reduced and maintained vaginal $\mathrm{pH}$ and the Nugent score and

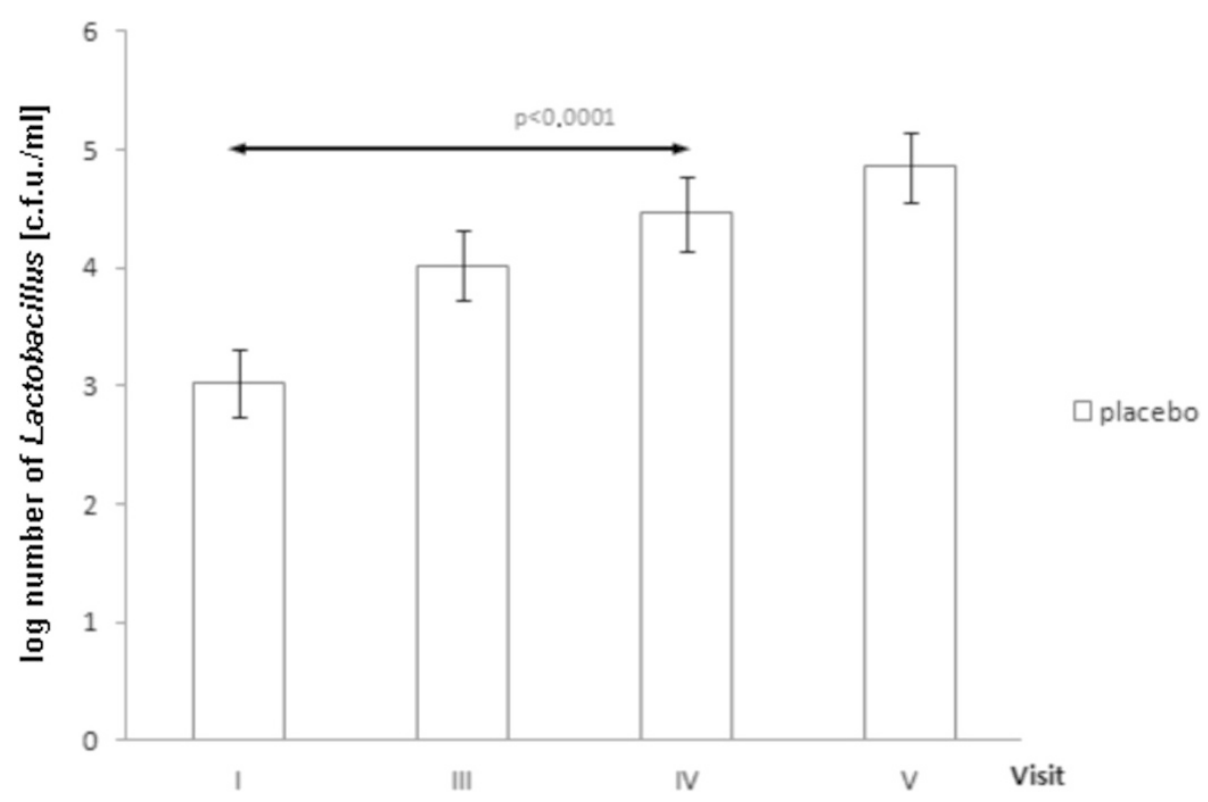

Fig. 7 Effect of placebo application on total numbers of vaginal lactobacilli during consecutive visits 
stimulated an increase in the Lactobacillus count in vaginal flora during standard treatment, thereby helping to sustain the effect of BV/AV treatment. This study demonstrated the safety and efficacy of prOVag in preventing $\mathrm{BV}$ recurrence, as this product contributes to the improvement of clinical and microbiological parameters, thereby lengthening the remission period.

\section{Additional file}

Additional file 1: Consort 2010 checklist of information to include when reporting a randomised trial [33]*. (DOC $214 \mathrm{~kb})$

\section{Competing interests}

Authors AMC and MSS represent IBSS BIOMED S.A., the company which has sponsored the research and manufactures the product used in the trial.

\section{Authors' contributions}

$\mathrm{PBH}, \mathrm{AMC}, \mathrm{MS}$ were involved in the design of the study protocol; $\mathrm{PBH}$ wrote the manuscript and was microbiological expert; AT was responsible for data collection and edited the manuscript; MS contributed to the interpretation of findings; PA was responsible for statistical analysis; AJJ was study clinical expert and safety assessor, MSS was responsible for coordination of the study and was co-author of final report; AMC was the supervisor of the whole clinical project, GS was responsible for the study design. All authors approved the final version of the manuscript.

\section{Acknowledgments}

The authors would like to give special thanks to the study sponsor (IBSS BIOMED S.A.), study coordinator, investigators, and cooperating staff: Dr. Z.Chełmicki (coordinator), Dr. B.Golański, Dr. K.Klimczyk, Dr. K.WadowskaJaszczyńska, Dr. M.Bobrzyńska, Dr. T.Bieda, Dr. G.Bogutyn, and Dr. M.Opławski (principal investigators).

\section{Author details}

${ }^{1}$ Chair of Microbiology, Jagiellonian University Medical College, Krakow, Poland. ${ }^{2}$ Institute of Nature Conservation, Polish Academy of Sciences, Krakow, Poland. ${ }^{3}$ Clinical Department of Obstetrics and Gynecology, Central Clinical Hospital of Ministry of Interior and Administration, Warsaw, Poland. ${ }^{4}$ Department of Reproductive Health Research Institute of Mother and Child, Warsaw, Poland. ${ }^{5}$ IBSS BIOMED S.A, Krakow, Poland.

Received: 16 June 2014 Accepted: 6 October 2015

Published online: 03 December 2015

\section{References}

1. Allsworth JE, Peipert JF. Prevalence of bacterial vaginosis: 2001-2004 National Health and Nutrition Examination Survey data. Obstet Gynecol. 2007;109(1):114-20.

2. Li J, McCormick J, Bocking A, Reid G. Importance of vaginal microbes in reproductive health. Reprod Sci. 2012;19(3):235-42.

3. Donders GG. Definition and classification of abnormal vaginal flora. Best Pract Res Clin Obstet Gynaecol. 2007;21(3):355-73.

4. Ugwumadu A, Reid F, Hay P, Manyonda I. Natural history of bacterial vaginosis and intermediate flora in pregnancy and effect of oral clindamycin. Obstet Gynecol. 2004;104(1):114-9.

5. Leitich $\mathrm{H}$, Kiss $\mathrm{H}$. Asymptomatic bacterial vaginosis and intermediate flora as risk factors for adverse pregnancy outcome. Best Pract Res Clin Obstet Gynaecol. 2007;21(3):375-90.

6. Tempera G, Furneri PM. Management of aerobic vaginitis. Gynecol Obstet Invest. 2010;70(4):244-9.

7. Oduyebo OO, Anorlu Rl, Oqunsola FT. The effects of antimicrobial therapy on bacterial vaginosis in non-pregnant women. Cochrane Database Syst Rev. 2009;3:CD006055.

8. Bradshaw CS, Morton AN, Hocking J, Garland SM, Morris MB, Moss LM, et al. High recurrence rates of bacterial vaginosis over the course of
12 months after oral metronidazole therapy and factors associated with recurrence. J Infect Dis. 2006;193(11):1478-86.

9. Han C, Wu W, Fan A, Wang Y, Zhang H, Chu Z, et al. Diagnostic and therapeutic advancements for aerobic vaginitis. Arch Gynecol Obstet. 2015;291(2):251-7.

10. Larsson PG, Stray-Pedersen B, Ryttig KR, Larsen S. Human lactobacilli as supplementation of clindamycin to patients with bacterial vaginosis reduce the recurrence rate; a 6-month, double-blind, randomized, placebo-controlled study. BMC Womens Health. 2008:8:3.

11. Marcone V, Rocca G, Lichtner M, Calzolari E. Long-term vaginal administration of Lactobacillus rhamnosus as a complementary approach to management of bacterial vaginosis. Int J Gynaecol Obstet. 2010;110(3):223-6.

12. Ya W, Reifer C, Miller LE. Efficacy of vaginal probiotic capsules for recurrent bacterial vaginosis: a double-blind, randomized, placebo-controlled study. Am J Obstet Gynecol. 2010;203(2):120.e1-6.

13. Delia A, Morgante G, Rago G, Musacchio MC, Petraglia F, De Leo V. Effectiveness of oral administration of Lactobacillus paracasei subsp. paracasei F19 in association with vaginal suppositories of Lactobacillus acidofilus in the treatment of vaginosis and in the prevention of recurrent vaginitis. Minerva Ginecol. 2006;58(3):227-31.

14. Vujic G, Jajac Knez A, Despot Stefanovic V, Kuzmic Vrbanovic V: Efficacy of orally applied probiotic capsules for bacterial vaginosis and other vaginal infections: a double-blind, randomized, placebo-controlled study. Eur J Obstet Gynecol Reprod Biol 2013, [Epub ahead of print]

15. Reid G, Bruce AW, Fraser N, Heinemann C, Owen J, Henning B. Oral probiotics can resolve urogenital infections. FEMS Immunol Med Microbiol. 2001;30(1):49-52

16. Strus M, Brzychczy-Włoch M, Kochan P, Heczko P. Hydrogen peroxide produced by Lactobacillus species as a regulatory molecule for vaginal microflora. Med Dosw Mikrobiol. 2004;56(1):67-77 [Article in Polish]

17. Hay P. Recurrent bacterial vaginosis. Curr Opin Infect Dis. 2009;22(1):82-6.

18. Senok AC, Verstraelen H, Temmerman M, Botta GA. Probiotics for the treatment of bacterial vaginosis. Cochrane Database Syst Rev. 2009;4:CD006289.

19. Friedman LM, Furberg CD, DeMets DL. Fundamentals of Clinical Trials. 3rd ed. New York: Springer-Verlag, selected chapters; 1996.

20. Cook RL, Redondo-Lopez V, Schmitt C, Meriwether C, Sobel JD. Clinical, microbiological, and biochemical factors in recurrent bacterial vaginosis. J Clin Microbiol. 1992;30(4):870-7.

21. Blackwell AL, Fox AR, Philips I, Barlow D. Anaerobic vaginosis (nonspecific vaginitis): clinical, microbiological and therapeutic findings. Lancet. 1986;2:1379-82

22. Nugent RP, Krohn MA, Hillier SL. Reliability of diagnosing bacterial vaginosis is improved by a standardized method of gram stain interpretation. J Clin Microbiol. 1991;29(2):297-301.

23. Srinivasan S, Morgan MT, Liu C, Matsen FA, Hoffman NG, Fiedler $T L$, et al. More than meets the eye: associations of vaginal bacteria with gram stain morphotypes using molecular phylogenetic analysis. PLoS One. 2013;8(10):e78633

24. Leclercq R, Cantón R, Brown DF, Giske CG, Heisig P, MacGowan AP, et al. EUCAST expert rules in antimicrobial susceptibility testing. Clin Microbiol Infect. 2013:19(2):141-60.

25. van de Wijgert JH, Borgdorff $H$, Verhelst $R$, Crucitti T, Francis $S$, Verstraelen $H$, et al. The vaginal microbiota: what have we learned after a decade of molecular characterization? PLoS One. 2014;9(8):e105998.

26. Strus M, Chmielarczyk A, Kochan P, Adamski P, Chełmicki Z, Chełmicki A, et al. Studies on the effects of probiotic Lactobacillus mixture given orally on vaginal and rectal colonization and on parameters of vaginal health in women with intermediate vaginal flora. Eur J Obstet Gynecol Reprod Biol. 2012;163(2):210-5

27. Tomusiak A, Strus M, Heczko PB. Antibiotic resistance of Gardnerella vaginalis isolated from cases of bacterial vaginosis. Ginekol Pol. 2011;82(12):900-4 [Article in Polish]

28. Austin MN, Beigi RH, Meyn LA, Hillier SL. Microbiologic response to treatment of bacterial vaginosis with topical clindamycin or metronidazole. J Clin Microbiol. 2005:43(9):4492-7.

29. Mayer BT, Srinivasan S, Fiedler TL, Marrazzo JM, Fredricks DN, Schiffer JT. Rapid and profound shifts in the vaginal microbiota following antibiotic treatment for bacterial vaginosis. J Infect Dis. 2015. jiv079.

30. Aroutcheva A, Simoes JA, Shott S, Faro S. The inhibitory effect of clindamycin on Lactobacillus in vitro. Infect Dis Obstet Gynecol. 2001;9(4):239-44 
31. Eriksson K, Larsson PG, Nilsson M, Forsum U. Vaginal retention of locally administered clindamycin. APMIS. 2011;119(6):373-6.

32. Anukam K, Osazuwa E, Ahonkhai I, Ngwu M, Osemene G, Bruce AW, et al. Augmentation of antimicrobial metronidazole therapy of bacterial vaginosis with oral probiotic Lactobacillus rhamnosus GR-1 and Lactobacillus reuteri RC-14: randomized, double-blind, placebo controlled trial. Microbes Infect. 2006;8(6):1450-4

33. Schulz KF, Altman DG, Moher D for the CONSORT Group. CONSORT 2010

Statement: updated guidelines for reporting parallel group randomised trials. Ann Intern Med. 2010;152(11):726-732.

\section{Submit your next manuscript to BioMed Central} and take full advantage of:

- Convenient online submission

- Thorough peer review

- No space constraints or color figure charges

- Immediate publication on acceptance

- Inclusion in PubMed, CAS, Scopus and Google Scholar

- Research which is freely available for redistribution 